\title{
Photonic crystal laser based on Fano interference allows for ultrafast frequency modulation in the $\mathrm{THz}$ range
}

\author{
Rasmussen, Thorsten S.; Yu, Yi; Mørk, Jesper
}

\section{Published in:}

Proceedings of SPIE

Link to article, DOI:

$10.1117 / 12.2506236$

Publication date:

2019

Document Version

Publisher's PDF, also known as Version of record

Link back to DTU Orbit

Citation (APA):

Rasmussen, T. S., Yu, Y., \& Mørk, J. (2019). Photonic crystal laser based on Fano interference allows for ultrafast frequency modulation in the THz range. In A. A. Belyanin, \& P. M. Smowton (Eds.), Proceedings of SPIE (Vol. 10939). [109390A] SPIE - International Society for Optical Engineering. Proceedings of SPIE - The International Society for Optical Engineering Vol. 10939 https://doi.org/10.1117/12.2506236

\section{General rights}

Copyright and moral rights for the publications made accessible in the public portal are retained by the authors and/or other copyright owners and it is a condition of accessing publications that users recognise and abide by the legal requirements associated with these rights.

- Users may download and print one copy of any publication from the public portal for the purpose of private study or research.

- You may not further distribute the material or use it for any profit-making activity or commercial gain

- You may freely distribute the URL identifying the publication in the public portal 


\section{Photonic crystal laser based on Fano interference allows for ultrafast frequency modulation in the $\mathrm{THz}$ range}

Thorsten S. Rasmussen, Yi Yu, Jesper Mørk

Thorsten S. Rasmussen, Yi Yu, Jesper Mørk, "Photonic crystal laser based on Fano interference allows for ultrafast frequency modulation in the $\mathrm{THz}$ range," Proc. SPIE 10939, Novel In-Plane Semiconductor Lasers XVIII, 109390A (1 March 2019); doi: 10.1117/12.2506236

SPIE. Event: SPIE OPTO, 2019, San Francisco, California, United States 


\title{
Photonic crystal laser based on Fano interference allows for ultrafast frequency modulation in the $\mathrm{THz}$ range
}

\author{
Thorsten S. Rasmussen ${ }^{\mathrm{a}}$, Yi Yü ${ }^{\mathrm{a}}$, and Jesper Mørk \\ ${ }^{a}$ DTU Fotonik, Technical University of Denmark, Ørsteds Plads, DK-2800 Kgs. Lyngby, \\ Denmark
}

\begin{abstract}
Replacing a conventional mirror in a photonic crystal laser by one based on Fano interference leads to rich laser dynamics, including realisation of stable self-pulsing and potential for ultra-fast modulation. In particular, the narrowband Fano mirror guarantees single-mode operation and significantly alters the modulation response compared to Fabry-Perot lasers. In this work the small-signal response is analysed using a dynamical model based on coupled-mode theory and rate equations, which shows how the 3-dB bandwidth of the frequency modulation response may exceed tens of $\mathrm{THz}$, orders of magnitude larger than for conventional semiconductor lasers.
\end{abstract}

Keywords: Fano laser, semiconductor laser, photonic crystal laser, Fano resonance, ultra-fast laser, frequency modulation, photonic crystal

\section{INTRODUCTION}

In the face of the increasing data consumption and the significant challenges this brings, it is essential to improve the efficiency and speed of optical communication systems. As of today, these systems are facing an electrical bottleneck due to the large transmission losses of electrical connections, resulting in significant heat dissipation limitations of many devices. ${ }^{1}$ One potential solution to this is to replace electrical interconnects by optical, which requires conversion from the electrical domain to the optical. ${ }^{2}$ This can be done using semiconductor lasers, and in particular for on-chip operation, nanolasers. Photonic crystal lasers ${ }^{3}$ have emerged as promising candidates for on-chip operation due to many useful properties, such as electrical pumping, high efficiency and intrinsically small size, leading to a small energy consumption. Much progress has been made in this field as of late, in particular towards the crucial steps of achieving electrical pumping, ${ }^{4}$ integration on silicon ${ }^{5}$ and thresholdless lasing, ${ }^{6}$ as well as numerous advances using passive photonic crystal platforms. ${ }^{7}$

Recently a novel type of photonic crystal laser was suggested ${ }^{8}$ and experimentally realised, ${ }^{9}$ in which one of the laser mirrors is replaced by a Fano resonance. This Fano resonance is a consequence of interference between a continuum of modes in a line-defect waveguide and a discrete mode of a nearby point-defect, effectively leading to a narrowband and dispersive reflection of the laser mirror, as illustrated in figure 1. This dispersive mirror provides the laser with a number of desirable properties. Due to the narrow bandwidth the laser is exclusively single-mode with an approximately constant frequency, ${ }^{9}$ and it was observed that the laser may transition into a self-pulsing state when the active material extends throughout the membrane, which was subsequently explained by the nanocavity functioning as a saturable absorber mirror. ${ }^{10}$ Furthermore, it has been suggested that the frequency modulation (FM) bandwidth of the laser may exceed $1 \mathrm{THz},{ }^{8,11}$ which is orders of magnitude larger than conventional semiconductor lasers, as these are limited to tens of $\mathrm{GHz}$ by the intrinsic relaxation oscillations. ${ }^{12}$ The study of this unusually large FM bandwidth is the subject of this paper.

The paper is structured as follows: Section 2 presents finite-difference time-domain (FDTD) simulations of the Fano laser device, demonstrating the working principle. In section 3, a simpler theoretical model used to analyse the laser is briefly introduced. In section 4 a small-signal analysis of this dynamical model is carried out, and the FM response is constructed from this analysis. In section 5 the FM response is analysed, and an alternative, high-resolution travelling-wave model is used to confirm the findings of the ODE model.

Further author information: (Send correspondence to T.S.R.)

T.S.R.: E-mail: thsv@fotonik.dtu.dk

Novel In-Plane Semiconductor Lasers XVIII, edited by Alexey A. Belyanin,

Peter M. Smowton, Proc. of SPIE Vol. 10939, 109390A · @ 2019 SPIE

CCC code: $0277-786 X / 19 / \$ 18 \cdot$ doi: $10.1117 / 12.2506236$

Proc. of SPIE Vol. 10939 109390A-1 

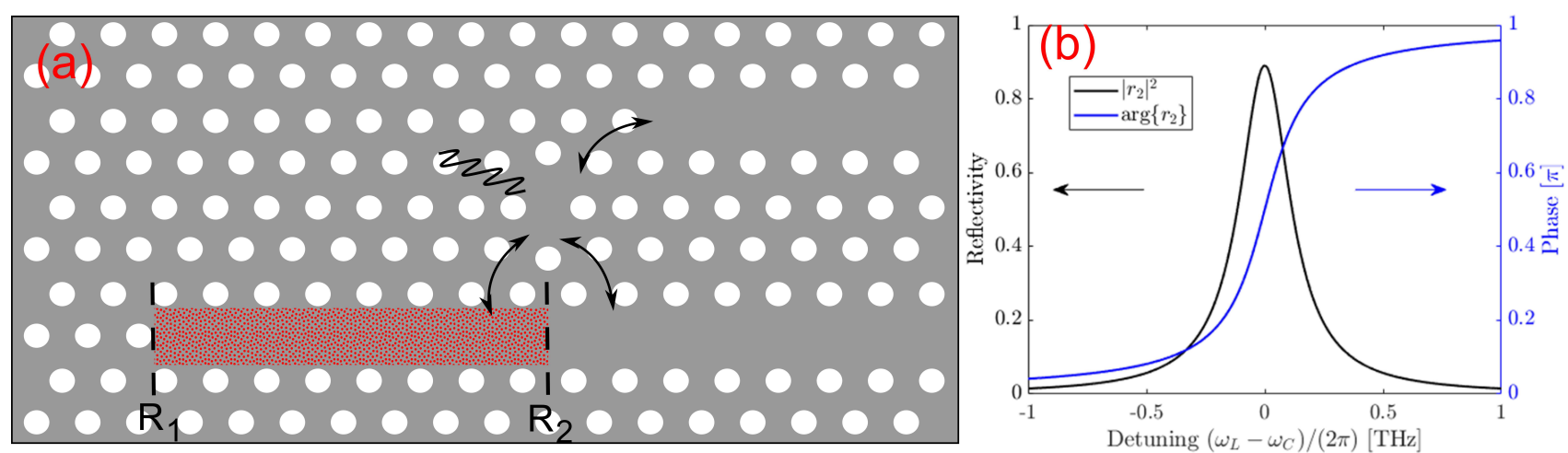

Figure 1: (a) Schematic of Fano laser structure. The grey area is a III-V semiconductor membrane, the white circles are air holes and the red area indicates the active region, where gain material is incorporated in the membrane. Arrows indicate coupling channels and dashed lines indicate effective mirrors. (b) Fano mirror intensity reflection and phase as function of the detuning between the incoming field and the nanocavity resonance frequency.

\section{FINITE-DIFFERENCE TIME-DOMAIN FANO LASER SIMULATIONS}

In order to demonstrate the Fano laser concept and gain fundamental insight into the device physics, full 3dimensional FDTD simulations have been carried out. In order to incorporate the active material to achieve lasing, the conventional, discretised Maxwell equations are coupled to rate equations for the level populations through the complex polarisation, as described in Ref. 13. In practice this is implemented using the commercial software FDTD Solutions by Lumerical inc, using a four-level two-electron model. It is interesting to note the limited number of FDTD-based laser calculations in the literature, but not surprising given the complexity and computational demands, and as such this type of calculation is interesting in itself. In Ref. 14 Cartar et al report in-depth investigations of conventional line-defect photonic crystal lasers using a 2D model with modified refractive index and structure size to mimic fully three-dimensional simulations, obtaining qualitative agreement with experimental results of Ref. 15. Here we report full 3-dimensional FDTD simulations of lasing action in a Fano laser structure.

Figure $2 \mathrm{a}$ shows the simulation setup from a top view of the dielectric membrane (InP). The slightly darker region is the active region, and the magenta arrow represents the optical pump for achieving population inversion. Figure $2 \mathrm{~b}$ shows the time evolution of the intensity of the electric field at the lasing wavelength, as well as the level populations $\left(N_{i, j}\right)$ at different spatial positions. The first index of $N_{i, j}$ refers to the upper (2) or lower (1) level of the lasing transition, while the second index refers to the spatial position, as indicated with the black numerals on figure 2a. Here one can observe how population inversion is gradually obtained: first close to the pump $\left(N_{21}\right.$ and $\left.N_{11}\right)$ and then at the second position and finally at the left end of the cavity, at which point full inversion is obtained and the lasing starts. This corresponds well with the time evolution of the field intensity, which starts growing as full inversion is obtained, displays a short oscillating transient and then reaches a steady-state with a corresponding narrowing of the optical spectrum. It is also worth noting that the laser oscillates at a frequency very close to the intrinsic resonance frequency of the nanocavity, as is expected from theory ${ }^{8}$ and as was also observed in experiments. ${ }^{9}$

\section{SIMPLER THEORETICAL MODEL}

While the FDTD simulations provide valuable insight into the fundamental properties of the device, they are time consuming and computationally demanding. As such, a simpler description of the laser is desired. One such description has previously been developed, for which the stationary properties of the laser are calculated based on a transmission line model, ${ }^{16}$ as described in Ref. 10. This leads to an oscillation condition similar to that of conventional Fabry-Perot lasers, but which includes a dispersive mirror, which in turn results in complicated behaviour when tuning both the length and the mirror resonance, as analysed in Refs. 8,10,11. 


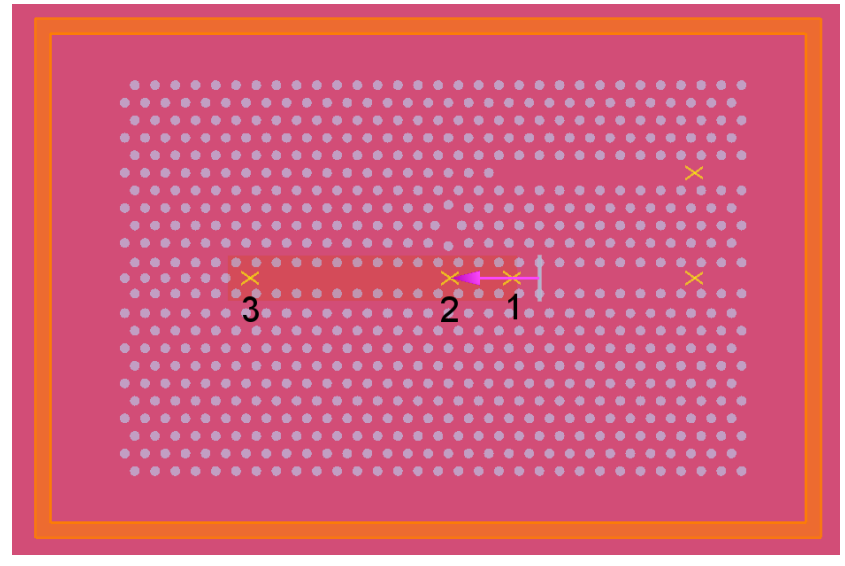

(a)

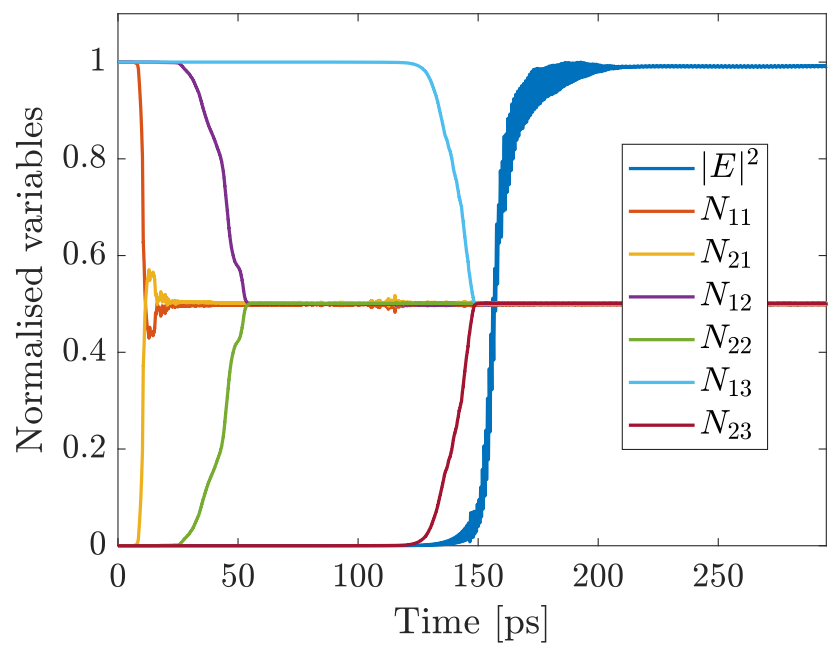

(b)

Figure 2: (a) FDTD simulation setup. The numbers correspond to the second index of $N_{i, j}$ in figure b. The pump source is the purple arrow and the slightly darker rectangle is the active region. The orange rectangle is an absorbing boundary condition (PML). (b) Time evolution of the level populations and the field intensity at the lasing wavelength (dark blue). The first index of $N$ indicates upper (2) or lower (1) level, and the second indicates the spatial position according to the numbers on figure $\mathbf{a}$.

By Taylor expansion and Fourier transform of this oscillation condition one can derive an equation for the field envelope in the laser cavity, which, when combined with rate equations for the field in the nanocavity ${ }^{17,18}$ and the carrier density ${ }^{12}$ in the waveguide, yields the following system of ordinary differential equations: ${ }^{11}$

$$
\begin{aligned}
\frac{\mathrm{d} A^{+}(t)}{\mathrm{d} t} & =\frac{1}{2}(1-i \alpha)\left(\Gamma v_{g} g_{N}\left(N-N_{0}\right)-\frac{1}{\tau_{p}}\right) A^{+}(t)+\gamma_{L}\left[\frac{\sqrt{\gamma_{c}} A_{c}(t)}{r_{2}\left(\omega_{L}, \omega_{c}\right)}-A^{+}(t)\right] \\
\frac{\mathrm{d} A_{c}(t)}{\mathrm{d} t} & =\left(-i \Delta \omega-\gamma_{T}\right) A_{c}(t)+i \sqrt{\gamma_{c}} A^{+}(t) \\
\frac{\mathrm{d} N(t)}{\mathrm{d} t} & =R_{P}-R(N)-\Gamma v_{g} g_{N}\left(N(t)-N_{0}\right) \sigma\left(\omega_{L}, \omega_{C}\right) \frac{\left|A^{+}(t)\right|^{2}}{V_{L C}}
\end{aligned}
$$

Here $A^{+}(t)$ is the envelope of the field in the laser cavity, while $A_{c}(t)$ is the field in the nanocavity, and $N(t)$ is the free carrier density in the active region. $\Gamma$ is the field confinement factor, $v_{g}=c / n_{g}$ is the group velocity, $\alpha$ is the linewidth enhancement factor, $g_{N}$ is the differential gain, $N_{0}$ is the transparency carrier density, $\tau_{p}$ is the photon lifetime, $\gamma_{L}=1 / \tau_{i n}$ is the inverse roundtrip time in the laser cavity, $\gamma_{c}$ is the coupling rate from the waveguide to the nanocavity, $r_{2}$ is the complex Fano reflection coefficient at the expansion point, $\Delta \omega$ is the detuning between the laser frequency and the nanocavity resonance frequency, and $\gamma_{T}$ is the total decay rate of the nanocavity field. Furthermore, $R_{P}$ is the pump rate in the active region, $R(N)=N / \tau_{s}$ is the recombination rate of the carriers, $V_{L C}$ is the volume of the laser cavity and $\sigma$ is a parameter relating the field strength and the photon number, $N_{P}$, as $N_{P}=\sigma_{s}\left|A^{+}(t)\right|^{2}$, see e.g. Ref. 10. The parameters used in the calculations in this paper are also identical to those therein. Based on the coupled-mode theory formulation employed, the output power in the through- and cross-ports is ${ }^{9}$

$$
\begin{aligned}
& P_{t}(t)=2 \epsilon_{0} n c\left|\sqrt{\gamma_{c}} A_{c}(t)-i A^{+}(t)\right|^{2} \\
& P_{x}(t)=2 \epsilon_{0} n c \gamma_{p}\left|A_{c}(t)\right|^{2}
\end{aligned}
$$

where the through-port is defined as the waveguide beyond the mirror plane indicated by $R_{2}$ in figure 1 , while the cross-port is the waveguide on the opposite side of the nanocavity.

In the limit where $\gamma_{T}$ is significantly larger than the other important time constants of the system (in particular 
$1 / \tau_{\text {in }}$ and $\left.1 / \tau_{s}\right)$, the nanocavity field may be adiabatically eliminated. This yields

$$
\begin{aligned}
A_{c}(t) & =\frac{i \sqrt{\gamma_{c}}}{i \delta_{c}+\gamma_{T}} A^{+}(t) \\
& =\frac{r_{2}\left(\omega_{L}, \omega_{c}\right)}{\sqrt{\gamma_{c}}} A^{+}(t)
\end{aligned}
$$

Inserting this into (1) and multiplying by the complex conjugate of $A^{+}(t)$ yields

$$
A^{+}(t)^{*} \frac{\mathrm{d} A^{+}(t)}{\mathrm{d} t}=\frac{1}{2}(1-i \alpha)\left(\Gamma v_{g} g_{N}\left(N-N_{0}\right)-\frac{1}{\tau_{p}}\right)\left|A^{+}(t)\right|^{2}
$$

Adding the complex conjugate of this equation and multiplying by $\sigma_{s}$ leads to

$$
\sigma_{s} \frac{\mathrm{d}\left|A^{+}(t)\right|^{2}}{\mathrm{~d} t}=\left(\Gamma v_{g} g_{N}\left(N-N_{0}\right)-\frac{1}{\tau_{p}}\right)\left|A^{+}(t)\right|^{2} \sigma_{s}
$$

which by the previously introduced definition $N_{p}=\sigma_{s}\left|A^{+}(t)\right|^{2}$ is equivalent to the conventional rate equation for the photon number of a Fabry-Perot semiconductor laser. ${ }^{12}$ In other words, when the mirror linewidth (given by $\gamma_{T}{ }^{8}$ ) is sufficiently broad, the Fano laser becomes equivalent to a conventional laser, as would also be expected intuitively, since the discerning feature of the Fano laser is the dispersive mirror.

\section{SMALL-SIGNAL ANALYSIS}

In order to study the modulation response of the laser, a small-signal analysis is employed. The first step is to separate the dynamical equations into amplitude and phase, and the resulting equations are then linearised in order to obtain a system of the form

$$
\dot{\vec{x}}=\mathbf{A} \vec{x}+\vec{F}
$$

where $\vec{x}$ is the vector of small signal amplitudes, i.e. $x=\left[\delta\left|A^{+}(t)\right|, \delta\left|A_{c}(t)\right|, \delta \phi^{+}, \delta \phi_{c}, \delta N\right]^{T}, \mathbf{A}$ is the Jacobian matrix and $\vec{F}$ is an externally applied forcing function, which represents e.g. modulation. The solution vector $\vec{x}$ describes the response to an external perturbation from a steady-state, and can be used to extract the IM and FM response. Due to the complicated form of the field in the throughport (cf. equation (4)), one must be careful in interpreting the result of solving equation (10), in particular for constructing the FM response. The solution vector contains the small-signal response amplitudes $\left[\delta\left|a^{+}\right|, \delta\left|a_{c}\right|, \delta \Phi^{+}, \delta \Phi_{c}, \delta N\right]$, and from this vector the full fields under harmonic modulation (with frequency $\omega$ ) can be constructed as

$$
\begin{aligned}
& A^{+}(t)=\left(\left|A_{s}^{+}\right|+\delta\left|a^{+}\right| e^{i \omega t}\right) \exp \left(i\left(\Phi_{s}^{+}+\delta \Phi^{+} e^{i \omega t}\right)\right) \\
& A_{c}(t)=\left(\left|A_{c, s}\right|+\delta\left|a_{c}\right| e^{i \omega t}\right) \exp \left(i\left(\Phi_{c, s}+\delta \Phi_{c} e^{i \omega t}\right)\right)
\end{aligned}
$$

Since the system dynamics only depend on the phase difference between the two fields, ${ }^{10} \Phi_{s}^{+}$can be set to zero, and then the non-negligible part of the total through-port field becomes

$$
A_{t}(t)=\sqrt{\gamma_{c}}\left|A_{c, s}\right| \exp \left[i\left(\Delta \Phi+\delta \Phi_{c} e^{i \omega t}\right)\right]-i\left|A_{s}^{+}\right| \exp \left[i \delta \Phi^{+} e^{i \omega t}\right]
$$

by neglecting terms related to the small amplitude variations $\delta\left|a^{+}\right|$and $\delta\left|a_{c}\right|$, while the cross-port response follows directly from $\delta \phi_{c}$. From this total field the frequency response can then be extracted as the maximum of the time derivative and normalised to the modulation amplitude. In the next section this approach is utilised to study the response to an external modulation of the nanocavity resonance frequency, which leads to frequency modulation of the laser. 


\section{FREQUENCY MODULATION RESPONSE}

Due to the dispersive nature of the Fano mirror (cf. figure $1 \mathrm{~b}$ ), the laser phase condition and thus laser frequency depends strongly on the resonance frequency of the nanocavity and the cavity length. This means that applying an external modulation of the nanocavity resonance, e.g. through electrical ${ }^{19}$ or optical ${ }^{20,21}$ means, results in a corresponding modulation of the laser frequency, so that the output waveguide field becomes frequency modulated. However, the intensity reflectivity also depends on the nanocavity resonance, meaning that a large-amplitude modulation will result in both frequency and intensity modulation, leading to complex Q-switching dynamics. ${ }^{8}$ If the modulation amplitude is small (compared to the mirror linewidth $\gamma_{T}$ ), however, and the laser is operated near zero detuning, the result is an almost pure FM signal. ${ }^{8,11}$ Figure 3 shows the FM response in the cross-port when operating near zero detuning, as calculated using the formalism of section 4 , with a modulation of the nanocavity resonance frequency with amplitude $\epsilon \gamma_{T}$, with $\epsilon=0.1$. Thus, the frequency response is normalised to this amplitude.

Generally the FM response can be divided into three different regimes, defined by the relation between the modulation frequency and the field decay constants, $\gamma_{T}$ and $\gamma_{L}$. This relationship governs the dynamics of the laser during the modulation process. In the regime of low modulation frequencies both decay rates are fast enough that the laser continues to uphold the roundtrip phase condition, meaning that the laser adjusts its frequency with an amplitude relative to the modulation given by the tuning characteristics from the oscillation condition (see Refs. 10,11). This explains why the normalised response is below unity for frequencies below $\min \left(\gamma_{T}, \gamma_{L}\right)$, and the asymptotic value is given by ${ }^{11}$

$$
\left|H\left(\omega_{m} \ll \min \left(\gamma_{T}, \gamma_{L}\right)\right)\right|=\frac{1}{1+\frac{\gamma_{T}}{\gamma_{L}}}
$$

where $H\left(\omega_{m}\right)$ is the FM transfer function. As such, this regime is essentially equivalent to adiabatic tuning of the laser wavelength through the nanocavity resonance. From these arguments it follows naturally that the upper limit of this regime is approximately given by $\omega_{m}<\min \left(\gamma_{T}, \gamma_{L}\right)$, as is also evident from figure 3. As the modulation frequency begins to approach the smaller of the decay constants, the laser can no longer follow the modulation in the conventional way. However, instead of the response dropping off, as one would intuitively expect for a conventional laser, it instead increases to unity when $\omega_{m} \gg \gamma_{T}+\gamma_{L}$. What happens in this case is that the roundtrip condition is fulfilled on average, while the frequency of the nanocavity field is modulated within a single roundtrip cycle through the adiabatic wavelength conversion mechanism, ${ }^{22}$ which can be as fast as a

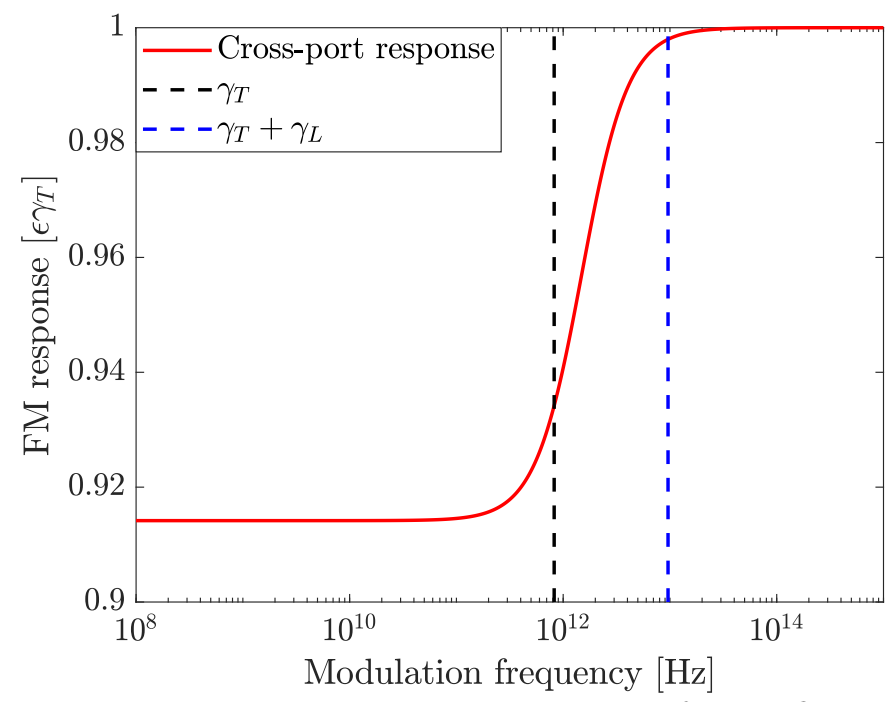

Figure 3: FM response normalised to the modulation amplitude $\epsilon \gamma_{T}$ for the field in the cross-port (red). The regime-defining decay constants $\gamma_{T}$ (dashed black) and $\gamma_{T}+\gamma_{L}$ (dashed blue) are indicated with vertical lines. For these parameters, $\gamma_{T} \ll \gamma_{L}$. 
few femtoseconds ${ }^{23}$ provided the modulation of the nanocavity index is spatially homogeneous, as assumed here. This frequency modulated nanocavity field is then essentially 'recycled' by the active laser cavity, corresponding to an independent modulation of the nanocavity field powered by the laser. This happens because the dynamics in this regime are too fast for any changes in field amplitude or carrier density to take place, as the modulation occurs within a single roundtrip. Crucially this means that a pure FM signal can potentially be transmitted with bandwidths orders of magnitude larger than for conventional lasers, provided one can generate the modulation of the nanocavity resonance, eliminating the conventional relaxation oscillation-imposed limitation.

The case for the through-port field is more complicated, in particular because the equation for the L-cavity field is a lumped model for which the temporal resolution is limited to the round-trip time due to the Taylor expansion used in the derivation. This means that the response of the forward field, $A^{+}(t)$, falls off as the modulation frequency approaches the round-trip time (see Refs. 8,11), and as such, a model with improved time resolution is necessary to correctly describe the through-port response, cf. equation (4).

\subsection{Iterative model}

One such improved model may be constructed by starting from an iterative travelling wave model for the electric field in the laser cavity, as in Ref. 24. This equation takes the following form:

$$
A^{+}\left(t+\tau_{i n}\right)=r_{L S} \exp \left[\frac{1}{2}(1-i \alpha) \tau_{i n} \Gamma v_{g} g_{N}\left(N-N_{s}\right)\right] \sqrt{\gamma_{c}} A_{c}(t)
$$

Here $r_{L S}=1 / r_{2}\left(\omega_{s}, N_{s}\right)$, where $\left(\omega_{s}, N_{s}\right)$ represents a steady-state solution of the system. This factor essentially represents the gain and phase acquired in propagation from the right mirror to the left and back again in steady-state, so that $r_{L S} r_{2}\left(\omega_{s}, N_{s}\right)=1$, fulfilling the oscillation condition. Numerically, this can conveniently implemented in a fully iterative scheme, by approximating the carrier density time evolution as

$$
N(t+\tau)=N(t)+\tau \frac{\mathrm{d} N}{\mathrm{~d} t}
$$

which is valid as the carrier density varies slowly on the scale of the roundtrip time. The nanocavity field is evolved by using the analytical solution to (2), which exists in closed form when the input $A^{+}(t)$ is constant over the time step of the solution. At this point, however, the temporal resolution is still limited by the round-trip time, $\tau_{i n}$, and the discretisation error for the nanocavity field may be notable due to the relatively long time step $(\approx 120 \mathrm{fs})$. In order to improve on this issue, the evolution equation is then discretised by subdividing the left- and right-propagating field along a number of nodes in the L-cavity and distributing the gain and expansion point gain $\left(r_{L S}\right)$, as illustrated in figure 4 . The field is propagated from node to node as

$$
\left.A_{n+1}^{+}=r_{L S}^{(\Delta L /(2 L))} \exp [G(N) \Delta L /(2 L)]\right) A_{n}^{+}
$$

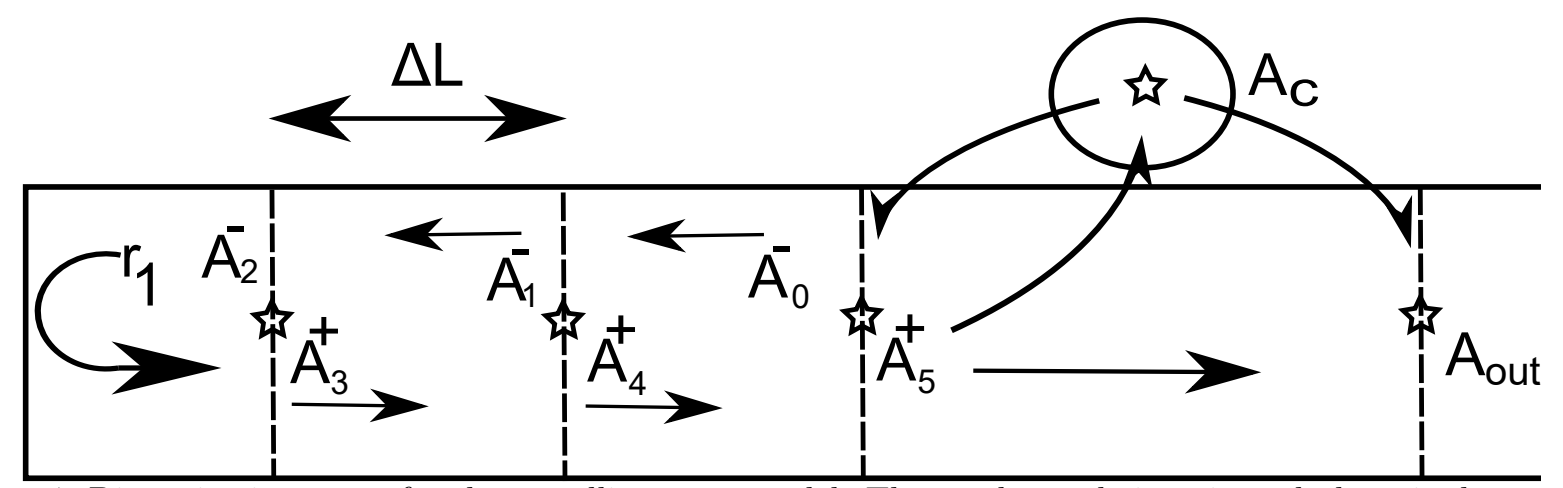

Figure 4: Discretisation setup for the travelling wave model. The total roundtrip gain and phase is the same as for the single-plane model, and the steady-state is identical, but the time resolution is dramatically improved, allowing for study of ultra-fast modulation. 


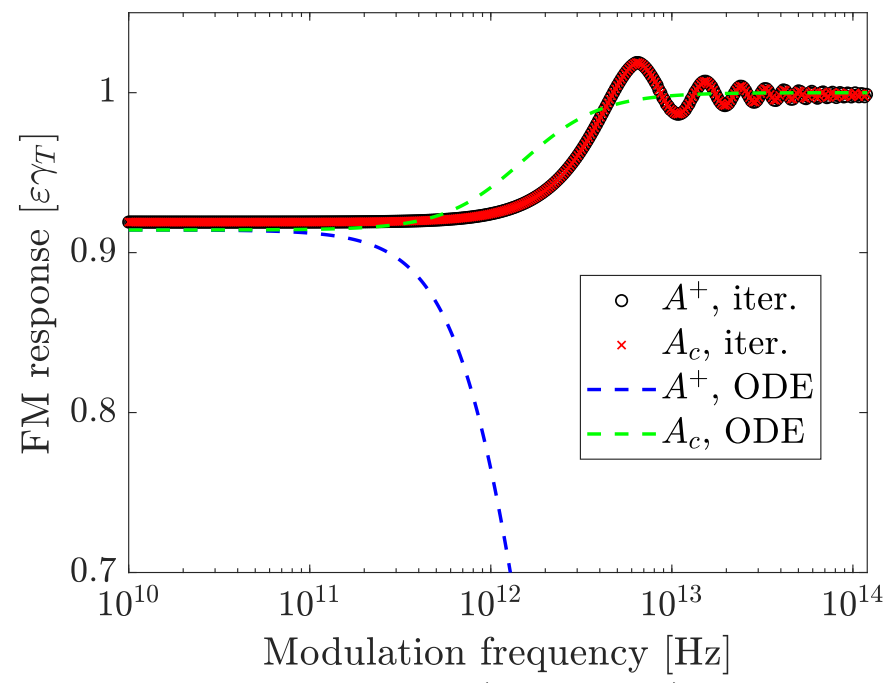

Figure 5: Comparison of FM response for the ODE model (dashed lines) and the travelling wave model (red and black markers). When the limitation of the lumped round-trip time model is removed, the forward field $\left(A^{+}\right)$ matches the backward field (proportional to $A_{c}$ ), as expected. Interestingly there are also some oscillations in the response in the ultra-high frequency regime, which are attributed to beating with the L-cavity roundtrip time.

where $G(N)=(1-i \alpha) \Gamma g_{N}\left(N-N_{s}\right)$. Since the field propagates $2 L / \Delta L$ per round-trip, the total round-trip gain is the same as for the non-discretised version. In this way the temporal resolution is improved by keeping track of the field at more points in space, which translates directly to additional points in time. This improved temporal resolution then allows for resolving ultra-fast modulation of the laser frequency on a time scale only determined by the number of discretisation points, with femtosecond resolution being readily obtained with memory available on a regular workstation.

Calculating the FM response directly using this model, by applying a modulation to the resonance frequency in the equation for the nanocavity field, yields the results shown in figure 5. Here the black circles and red crosses represent the FM response from the discretised iterative model, while the dashed lines are the same results but from the ODE model. It can be seen that removing the round-trip time limitation results in the forward-propagating field $\left(A^{+}(t)\right)$ matching the backward propagating field $\left(\sqrt{\gamma_{c}} A_{c}(t)\right)$, as is expected, since the only change between them is a reflection at the left mirror (red and black curves coincide). One can also observe how the travelling wave model response shows oscillations, beginning around $\gamma_{L}$. These are attributed to a beating effect between the modulation frequency and the roundtrip time, since the peaks are spaced by exactly $\gamma_{L}$ and only start once the modulation frequency exceeds the inverse roundtrip time. Furthermore, these oscillations converge as the modulation frequency becomes much larger than the roundtrip time, because this beating effect is 'washed out' by the increasingly large number of modulation cycles per roundtrip. It is also natural that these oscillations are not evident in the ODE model, since they require the coherent, time-resolved interaction of the L-cavity field, which is not well-described beyond $\gamma_{L}$ in the ODE model.

While it is still an open question how to realise efficient, high-speed modulation of the nanocavity resonance frequency, these calculations suggest that if such a modulation can be realised, then data can be transmitted at rates hugely exceeding the relaxation oscillation limitation of conventional Fabry-Perot laser based systems. ${ }^{25}$ Thus, the essential point is that the laser itself is not intrinsically limited to GHz modulation frequencies, unlike conventional Fabry-Perot lasers, so that the challenge of high-speed on-chip data transfer is reduced from designing the laser to providing the modulation, and that the intrinsic speed of the laser (and thus potential data rate) is essentially only limited by the time scale of the adiabatic wavelength conversion process, which is extremely fast (few fs ${ }^{23}$ ). 


\section{CONCLUSION AND SCOPE}

The photonic crystal Fano laser was briefly introduced and described, and full 3D FDTD simulations of laser action in the device were reported. It was explained how to calculate the small-signal response of the laser, and the frequency-modulation response was analysed in detail, demonstrating three different regimes of operation, governed by the characteristic decay times of the fields in the nanocavity and laser cavity. In order to properly resolve ultra-fast modulation $(>\mathrm{THz})$, an iterative travelling wave model was developed, and it was shown how the FM bandwidth of the Fano laser exceeds conventional lasers by orders of magnitude, due to adiabatic wavelength conversion of the nanocavity field allowing for generation of ultra-fast and very pure FM signals by modulation of the nanocavity resonance frequency.

\section{ACKNOWLEDGMENTS}

The authors acknowledge financial support from Villum Fonden through the NATEC center of excellence.

\section{REFERENCES}

[1] Garimella, S. V., Fleischer, A. S., Murthy, J. Y., Keshavarzi, A., Prasher, R., Patel, C., Bhavnani, S. H., Venkatasubramanian, R., Mahajan, R., Joshi, Y., Sammakia, B., Myers, B. A., Chorosinski, L., Baelmans, M., Sathyamurthy, P., and Raad, P. E., "Thermal challenges in next-generation electronic systems," IEEE Transactions on Components and Packaging Technologies 31, 801-815 (Dec 2008).

[2] Miller, D. A. B., "Device requirements for optical interconnects to silicon chips," Proceedings of the IEEE 97, 1166-1185 (July 2009).

[3] Painter, O., Lee, R. K., Scherer, A., Yariv, A., O’Brien, J. D., Dapkus, P. D., and Kim, I., "Two-dimensional photonic band-gap defect mode laser," Science 284(5421), 1819-1821 (1999).

[4] Matsuo, S., Sato, T., Takeda, K., Shinya, A., Nozaki, K., Taniyama, H., Notomi, M., Hasebe, K., and Kakitsuka, T., "Ultralow operating energy electrically driven photonic crystal lasers," IEEE Journal of Selected Topics in Quantum Electronics 19, 4900311-4900311 (July 2013).

[5] Crosnier, G., Sanchez, D., Bouchoule, S., Monnier, P., Beaudoin, G., Sagnes, I., Raj, R., and Raineri, F., "Hybrid indium phosphide-on-silicon nanolaser diode," Nature Photonics 11 (Apr 2017).

[6] Ota, Y., Kakuda, M., Watanabe, K., Iwamoto, S., and Arakawa, Y., "Thresholdless quantum dot nanolaser," Opt. Express 25, 19981-19994 (Aug 2017).

[7] Notomi, M., Shinya, A., Nozaki, K., Tanabe, T., Matsuo, S., Kuramochi, E., Sato, T., Taniyama, H., and Sumikura, H., "Low-power nanophotonic devices based on photonic crystals towards dense photonic network on chip," IET Circuits, Devices Systems 5, 84-93 (March 2011).

[8] Mork, J., Chen, Y., and Heuck, M., "Photonic crystal fano laser: Terahertz modulation and ultrashort pulse generation," Phys. Rev. Lett. 113, 163901 (Oct 2014).

[9] Yu, Y., Xue, W., Semenova, E., Yvind, K., and Mork, J., "Demonstration of a self-pulsing photonic crystal fano laser," Nat Photon 11, 81-84 (Feb 2017). Letter.

[10] Rasmussen, T. S., Yu, Y., and Mork, J., "Theory of self-pulsing in photonic crystal fano lasers," Laser $\&$ Photonics Reviews 11(5), 1700089 (2017).

[11] Rasmussen, T. S., Yu, Y., and Mork, J., "Modes, stability, and small-signal response of photonic crystal fano lasers," Opt. Express 26, 16365-16376 (Jun 2018).

[12] Coldren, L. and Corzine, S., [Diode Lasers and Photonic Integrated Circuits], Wiley (1st edition, Wiley 1995).

[13] Chang, S.-H. and Taflove, A., "Finite-difference time-domain model of lasing action in a four-level twoelectron atomic system," Opt. Express 12, 3827-3833 (Aug 2004).

[14] Cartar, W., Mørk, J., and Hughes, S., "Self-consistent maxwell-bloch model of quantum-dot photoniccrystal-cavity lasers," Phys. Rev. A 96, 023859 (Aug 2017).

[15] Xue, W., Yu, Y., Ottaviano, L., Chen, Y., Semenova, E., Yvind, K., and Mork, J., "Threshold characteristics of slow-light photonic crystal lasers," Phys. Rev. Lett. 116, 063901 (Feb 2016). 
[16] Tromborg, B., Olesen, H., Pan, X., and Saito, S., "Transmission line description of optical feedback and injection locking for fabry-perot and dfb lasers," Quantum Electronics, IEEE Journal of 23, 1875-1889 (Nov 1987).

[17] Fan, S., Suh, W., and Joannopoulos, J. D., "Temporal coupled-mode theory for the fano resonance in optical resonators," J. Opt. Soc. Am. A 20, 569-572 (Mar 2003).

[18] Kristensen, P. T., de Lasson, J. R., Heuck, M., Gregersen, N., and Mork, J., "On the theory of coupled modes in optical cavity-waveguide structures," Journal of Lightwave Technology 35, 4247-4259 (Oct 2017).

[19] Haret, L.-D., Checoury, X., Bayle, F., Cazier, N., Boucaud, P., Combrié, S., and de Rossi, A., "Schottky msm junctions for carrier depletion in silicon photonic crystal microcavities," Opt. Express 21, 10324-10334 (Apr 2013).

[20] Koenderink, A. F., Kafesaki, M., Buchler, B. C., and Sandoghdar, V., "Controlling the resonance of a photonic crystal microcavity by a near-field probe," Phys. Rev. Lett. 95, 153904 (Oct 2005).

[21] Tanabe, T., Notomi, M., Taniyama, H., and Kuramochi, E., "Dynamic release of trapped light from an ultrahigh- $q$ nanocavity via adiabatic frequency tuning," Phys. Rev. Lett. 102, 043907 (Jan 2009).

[22] Daniel, B. A., Maywar, D. N., and Agrawal, G. P., "Dynamic mode theory of optical resonators undergoing refractive index changes," J. Opt. Soc. Am. B 28, 2207-2215 (Sep 2011).

[23] Notomi, M. and Mitsugi, S., "Wavelength conversion via dynamic refractive index tuning of a cavity," Phys. Rev. A 73, 051803 (May 2006).

[24] Mork, J., Tromborg, B., and Christiansen, P. L., "Bistability and low-frequency fluctuations in semiconductor lasers with optical feedback: a theoretical analysis," IEEE Journal of Quantum Electronics 24, 123-133 (Feb 1988).

[25] Zali, A. R., Moravvej-Farshi, M. K., Yu, Y., and Mork, J., "Small and large signal analysis of photonic crystal fano laser," J. Lightwave Technol. 36, 5611-5616 (Dec 2018). 